\title{
EFECTO DE LA RADIACIÓN GAMMA SOBRE LAS CARACTERÍSTICAS FÍSICO - QUIIMICAS, SENSORIALES Y MICROBIOLÓGICAS EN PÁPRIKA EN POLVO \\ (Capsicum annuum L.)
}

\author{
EFFECT OF GAMMA RADIATION ON THE PHYSICO-CHEMICAL, \\ MICROBIOLOGICAL CHARACTERISTICS AND SENSORY ANALYSIS OF \\ PAPRIKA POWDER (Capsicum annuum L.)
}

\section{RESUMEN}

\author{
Johnny Vargas R. ${ }^{a}$
}

El Perú es el primer exportador mundial de páprika, especia susceptible a la contaminación microbiana. El objetivo fue evaluar las características físico-químicas y sensoriales en páprika en polvo irradiadas a diferentes dosis de radiación gamma, con la finalidad de determinar la dosis mínima que reduzca la población microbiana a las especificaciones. Muestras de $500 \mathrm{~g}$ fueron irradiadas en el equipo Gammacell 220 (0; 2; 4 y $7 \mathrm{kGy}$ ). La tasa de dosis inicial fue de 5.19 kGy/h. Se realizaron los siguientes análisis: Físico-químico (Humedad, cenizas totales, cenizas insolubles en $\mathrm{HCl}$, extracto etéreo total, almidón, fibra cruda, índice de yodo y color asta). Evaluación sensorial ( Test descriptivo). Análisis microbiológico (Aerobios mesófilos, Salmonella/Shigella, Coliformes totales, Eschericha coli, Clostridium sulfito reductor, hongos y levaduras). Los métodos fueron los recomendados por la FDA edición 6ta, la AOAC (1984) y las NTP. A las diferentes dosis ensayadas, no se encontraron diferencias en los análisis físico-químicos y sensoriales. La dosis mínima seleccionada fue de $7 \mathrm{kGy}$ que reduce la población de microorganismos aerobios mesófilos en 5 ciclos logarítmicos de $3,7 \times 10^{6}$ a $2,5 \times 10 \mathrm{UFC} / \mathrm{g}$. Dosis de $4 \mathrm{kGy}$ fue suficiente para reducir la población de hongos en 3 ciclos logarítmicos de $5,0 \times 10^{3}$ a $<10 \mathrm{UFC} / \mathrm{g}$, cumpliendo con las especificaciones requeridas.

Palabras clave: Radiación gamma, páprika, dosis mínima, población microbiana

\section{ABSTRACT}

Peru is the world's leading exporter of paprika, spice susceptible to microbial contamination. The objective was to evaluate the physico-chemical and sensory paprika powder irradiated at different doses of gamma radiation, with the purpose of determining the lowest dose that reduces the microbial population to the specifications. $500 \mathrm{~g}$ samples were irradiated in the team Gammacell 220 (0, 2, 4 and $7 \mathrm{kGy}$ ). The initial dose rate was $5.19 \mathrm{kGy} / \mathrm{h}$. The samples were analized: Physical and chemical (moisture, total ash, $\mathrm{HCl}$-insoluble ash, ether extract, total starch, crude fiber, iodine value and ASTA color). Sensory evaluation (Test descriptive). Microbiological analysis (aerobic mesophiles, Salmonella / Shigella, total coliforms, Eschericha coli, Clostridium sulfite reducer, fungi and yeasts). The methods were those recommended by the 6th edition FDA, AOAC (1984) and NTP. At different doses tested, there were no differences in the physical-chemical analysis and sensory. The minimum dose of $7 \mathrm{kGy}$ was selected to reduce the population of mesophilic aerobic microorganisms in 5 log cycles of $3.7 \times 10^{6} \mathrm{CFU} / \mathrm{g}$ to $2.5 \times 10 \mathrm{CFU} / \mathrm{g} .4 \mathrm{kGy}$ dose was suficinte to reduce the population of fungi in $3 \mathrm{log}$ cycles of $5,0 \times 10^{3}$ to $<10 \mathrm{CFU} / \mathrm{g}$. according to the required specifications.

Key words: Gamma radiation, paprika, minimun dose, microbial population.

\section{INTRODUCCIÓN}

Las especias generalmente se encuentran contaminadas por diversos microorganismos, la páprika es susceptible a la contaminación de bacterias y hongos, desde la cosecha, procesamiento y almacenamiento.

Las especias contienen frecuentemente gran número de microorganismos causantes de descomposición o de enfermedades cuando se incorporan a los alimentos, la población microbiana puede sobrepasar el nivel de $10^{8}$ UFC/g (cien millones de unidades de microorganismos formadores de colonias). La carga de hongos puede llegar a veces a $10^{5}-10^{6} \mathrm{UFC} / \mathrm{g}$. [1] Algunos de sus usos como es el caso de la páprika están dirigidos a la elaboración de embutidos, formulaciones a base de vegetales, pescado, productos de panadería, sopas y salsas preparadas. [2] Se debe tener en cuenta, que la páprika en polvo también es usada, tal como ofrecida, sin ningún

\footnotetext{
${ }^{a}$ Dirección de Aplicaciones Instituto Peruano de Energía Nuclear
}

tratamiento térmico posterior, como es el caso en el consumo de pizzas y productos de snack bar, lo cual es riesgoso para el consumidor.[3].

El páprika se empezó a utilizar como especia por su capacidad de modificar el color de los alimentos, mejorar el aspecto y conferir características organolépticas particulares.

Actualmente se la usa no solo como sazonador, si no también como colorante en gran variedad de productos, entre los cuales se pueden incluir la industria láctea (quesos, mantequillas, etc.), industria de los piensos (avicultura, piscicultura, ganadería, etc.), industria conservera (vegetales, hidrobiológicos y cárnicos), industria de panificación (pasteles, galletas, etc.), gelatinas, pudines, embutidos y otros productos cárnicos, salsas, sopas, mayonesa, condimentos, bebidas refrescantes, congelados, polvos, jarabes, concentrados, industria cosmética (lápices labiales, polvos faciales, etc.). 
Frente a políticas de preservación del medio ambiente y las tendencias al uso de colorantes naturales a nivel mundial en reemplazo de los colorantes artificiales el consumo de páprika continúa en aumento ligado también a los desarrollos en la tecnología de los colorantes naturales como son los extractos y concentrados hidrosolubles y liposolubles. El contenido de carotenoides del páprika pueden sustituir los colorantes Amarillo \#5 y \#6, a la Tartrazina y al Subset Yellow.[4]

Por otro lado, La creciente demanda internacional por saborizantes y colorantes naturales permitió a la páprika consolidarse como uno de los principales productos de la agroexportación peruana, ubicando al país como el principal exportador mundial de la hortaliza: en 2007 el Perú exportó un total de US\$ 93.8 millones siendo el principal abastecedor del mercado de EE.UU. (con cerca del $70 \%$ del total de sus importaciones) y de la Unión Europea (con cerca del $30 \%$ del total de sus importaciones. [5]

Los objetivos de la irradiación en páprika en polvo irradiado fueron:

Reducir la población microbiana y hongos al nivel de las especificaciones sin alterar significativamente sus características físico-químicas y sensoriales.

No se utiliza la irradiación para la preservación de estos productos. La preservación se obtiene mediante el empaque, almacenamiento adecuado y secado apropiado.

\section{Características del Producto a Irradiar}

El proceso de irradiación se aplica a estos productos en las formas en las cuales normalmente se preparan para su uso y comercialización. Las consideraciones usuales tomadas en cuenta son la calidad y el uso de las buenas prácticas de producción. La irradiación puede ser utilizada para corregir deficiencias de calidad. Deberá evitarse la excesiva contaminación con microorganismos antes de la irradiación. [6]

\section{Dosis}

La unidad de dosis absorbida es el gray (Gy), que viene a ser la energía absorbida de un Joule por $\mathrm{Kg}$. de materia irradiada:

$$
\mathrm{Gy}=\text { Joule/Kg. }
$$

\section{MATERIALES Y MÉTODOS}

Las muestras corresponden a una Industria Nacional. Los productos fueron irradiados en el Equipo de Irradiación Gammacell 220 previa dosimetría Fricke y de acuerdo a la densidad y geometría del producto nos permitió hallar la tasa de dosis, para calcular los tiempos de exposición a la radiación gamma, para evaluar las dosis seleccionadas. Para la dosimetría se utilizó el método ASTM E 1026 1995 Practice for Using the Fricke Reference Standard Dosimetry System

Los métodos empleados en los análisis Físico-Químico y las especificaciones para páprika en polvo fueron consultados de la AOAC y de las Normas Técnicas Nacionales e Internacionales.
Para almidón se utilizó el método de Lane-Eynon. Los métodos empleados en los análisis microbiológicos fueron realizados de acuerdo a lo que señala la FDA (6ta Edición).

La evaluación sensorial se realizó mediante la prueba de comparación que permite encontrar diferencias entre dos o más muestras cuando estas son presentadas a los panelistas, quienes dan sus respuestas o calificaciones a través de términos descriptivos. Con el objeto de obtener una medición cuantitativa se puntuaron los resultados sobre una escala hedónica de 1 a 5 puntos, donde:

$\begin{array}{ll}\text { Excelente } & -5 \text { puntos } \\ \text { Bueno } & -4 \text { puntos } \\ \text { Satisfactorio } & -3 \text { puntos } \\ \text { Regular } & -2 \text { puntos } \\ \text { Malo } & -1 \text { punto }\end{array}$

Intervinieron en el desarrollo de esta prueba 5 panelistas.

\section{RESULTADOS Y DISCUSIÓN}

Tabla 1. Análisis microbiológico en páprika

\begin{tabular}{|c|c|c|c|c|}
\hline DETERMINACIONES & Control & $2 \mathrm{kGy}$ & 4 kGy & 7 kGy \\
\hline $\begin{array}{l}\text { R.Aerobios mesófilos UFC/g } \\
\text { Recuento de Hongos UFC/g } \\
\text { Recuento Levaduras UFC/g } \\
\text { R. Hifas (Met. Howard)(\%) } \\
\text { D. Salmonella/Shigella }(25 \mathrm{~g}) \\
\text { Coliformes totales (NMP/g) } \\
\text { Detección E. coli (en } 25 \mathrm{~g}) \\
\text { R Clostridium sulfito } \mathrm{r} \text { UFC/g }\end{array}$ & $\begin{array}{c}3,7 \times 10^{6} \\
5,0 \times 10^{3} \\
<10 \\
5 \\
\text { Ausente } \\
<0,30 \\
\text { Ausente } \\
10\end{array}$ & $\begin{array}{c}2,6 \times 10^{5} \\
5,0 \times 10^{2} \\
<10 \\
2 \\
\text { Ausente } \\
<0,30 \\
\text { Ausente } \\
<10\end{array}$ & 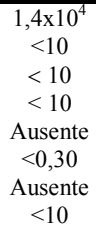 & $\begin{array}{c}2,5 \times 10 \\
\quad<10 \\
<10 \\
<10 \\
\text { Ausente } \\
<0,30 \\
\text { Ausente } \\
<10\end{array}$ \\
\hline
\end{tabular}

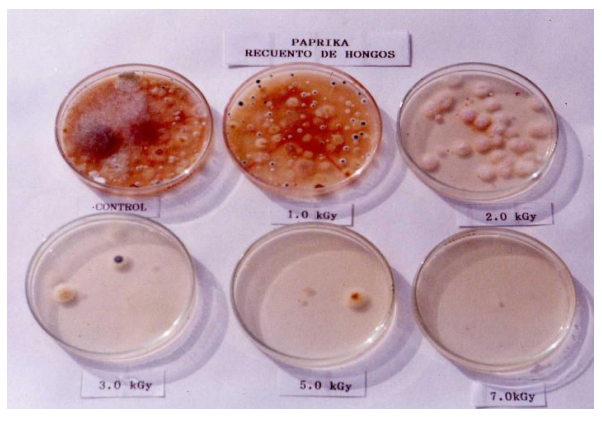

Figura 1: Recuento de hongos en páprika

Podemos apreciar en la tabla 1 que la páprika en polvo presenta una elevada contaminación de aerobios mesófilos de $3,7 \times 10^{6} \mathrm{UFC} / \mathrm{g}$ y al aplicarse la dosis de 7 kGy se logra reducir 5 ciclos logarítmicos a 2,5x10 UFC/g.

En lo que respecta a los hongos, podemos notar que es suficiente una dosis de $4 \mathrm{kGy}$ para reducir completamente la presencia de hongos en 3 ciclos logarítmicos, de $5,0 \times 10^{3} \mathrm{UFC} / \mathrm{g}$ a $<10 \mathrm{UFC} / \mathrm{g}$. También se encontró hifas en un $5 \%$ eliminándose a la dosis de $4 \mathrm{kGy}$.

Con la dosis de 2 kGy controlamos el recuento del Clostridium sulfito reductor de $10 \mathrm{UFC} / g$ a $<10 \mathrm{UFC} / \mathrm{g}$. Según la Norma ITINTEC [7], las especificaciones microbiológicas son en aerobios mesófilos máximo $1 \times 10^{5}$ UFC/g, en recuento de hongos, levaduras y Clostridium sulfito reductor máximo $10 \mathrm{UFC/g}$, e hifas máximo $40 \%$ 
En la muestra de páprika analizada no se encontró levaduras, Salmonella,/Shiguella, Coliformes totales, Clostridium sulfito reductor ni Escherichia coli.

Tabla 2. Análisis físico-químico en páprika

\begin{tabular}{lcccc}
\hline DETERMINACIONES & Control & 2 kGy & 4kGy & 7kGy \\
\hline Humedad (\%) & & & & \\
Cenizas totales (\%) & 15,54 & 15,69 & 15,99 & 15,99 \\
Cenizas insolubles & 5,87 & 5,76 & 5,82 & 5,99 \\
HCl\% & 0,35 & 0,37 & 0,36 & 0,36 \\
Extracto etéreo total & 13,94 & 13,70 & 14,30 & 13,76 \\
$(\%)$ & $\mathrm{N} \mathrm{Dt}$ & $\mathrm{N} \mathrm{Dt}$ & $\mathrm{N} \mathrm{Dt}$ & $\mathrm{N} \mathrm{Dt}$ \\
Almidón (\%) (*) & 20,06 & 20,21 & 20,2 & 20,12 \\
Fibra cruda (\%) & 97 & 92 & 91 & 94 \\
Índice de lodo & 141 & 145 & 142 & 137 \\
Color Asta & & & & \\
& & & &
\end{tabular}

$\left({ }^{*}\right) \mathrm{N} \mathrm{Dt}=$ No Detectable

Tabla 3. Especificaciones de análisis químico en páprika

\begin{tabular}{|c|c|c|}
\hline $\begin{array}{l}\text { DETERMIN } \\
\text { ACIÓN }\end{array}$ & ESPECIFICACIÓN & REFERENCIA \\
\hline $\begin{array}{l}\text { Humedad } \\
\text { Cenizas totales } \\
\text { Ceniza insolubleHCl } \\
\text { Extracto etéreo total } \\
\text { Almidón } \\
\text { Fibra cruda } \\
\text { Índice de lodo } \\
\text { Color Asta }\end{array}$ & $\begin{array}{l}\text { Máximo } 11.0 \% \\
\text { Máximo } 8.0 \% \\
\text { Máximo } 1.25 \% \\
\text { Máximo } 15.0 \% \\
\text { Mínimo } 0.5 \% \\
\text { Máximo } 28.0 \% \\
\text { Máximo } 136 \\
\text { Mínimo } 120\end{array}$ & $\begin{array}{c}\text { N. ITINTEC 209.190, } 1982 \\
\text { N. ITINTEC 209.190, } 1982 \\
\text { N. ITINTEC 209.190, } 1982 \\
\text { Código Alimentario Argent. } \\
\text { N. ITINTEC 209.190, } 1982 \\
\text { N. ITINTEC 209.190, } 1982 \\
\text { N. Mexicana - F -001,1982 } \\
\text { Código Alimentario } \\
\text { Argentino 2008 }\end{array}$ \\
\hline
\end{tabular}

En lo que respecta a los análisis físico-químicos no hay diferencias a las diferentes dosis ensayadas y están dentro de las especificaciones de la tabla 3, excepto en el • porcentaje de humedad que exige un máximo de $11 \%$ y los resultados de la investigación arrojan resultados entre $15,54 \%$ para el control y $15,99 \%$ para la dosis de $7 \mathrm{kGy}$, sin influir en estos datos las dosis administradas. En el • análisis de almidón, la norma demanda un mínimo de $0.5 \%$, en las muestras ensayadas, para todos los tratamientos, no se detecto almidón, tanto en la muestra • control, como en las muestras irradiadas, por lo que el efecto de la radiación no tiene influencia, en esta comparación.

En los demás análisis realizados como en cenizas totales, no hay diferencias importantes, tenemos valores entre $5,76 \%$ a $5,99 \%$, siendo $8 \%$ el máximo requerido, al igual • en cenizas insolubles en $\mathrm{HCl}$ entre $0,35 \%$ del Control y $0,36 \%$ de la muestra irradiada a $7 \mathrm{kGy}$, siendo hasta $1.25 \%$ el aceptado. En el extracto etereo total o grasa no • hubo alteración a las diferentes dosis ensayadas entre $13,70 \%$ y $14,30 \%$, contra el máximo del $15 \%$, también en fibra cruda se mantuvo constante entre 20,06 y 20,21 , debajo del máximo de $28 \%$.

En el índice de yodo que nos sirve para medir el grado de saturación de la grasa o aceite los valores arrojados son similares oscilando entre 91 a la dosis de 4 kGy y 97 de la muestra control.
En los análisis de color ASTA (American Spice Trade Association), de suma importancia para la exportación, no se encontraron diferencias significativas a las dosis ensayadas, con valores de 137 a 141, comparados con la clasificación extra que permite un mínimo de 120 ASTA.[8]

Tabla 4. Análisis sensorial en páprika

\begin{tabular}{lcccc}
\hline Atributo & Control & 2 kGy & 4kGy & 7kGy \\
\hline Color & Rojo oscuro, & Rojo oscuro & Rojo oscuro & Rojo oscuro \\
Olor & $\begin{array}{c}\text { Aromático } \\
\text { Característico }\end{array}$ & $\begin{array}{c}\text { Aromático } \\
\text { Característico }\end{array}$ & $\begin{array}{c}\text { Aromático } \\
\text { Característico }\end{array}$ & $\begin{array}{c}\text { Aromático } \\
\text { Característico }\end{array}$ \\
Sabor & $\begin{array}{c}\text { Picante } \\
\text { Característico }\end{array}$ & $\begin{array}{c}\text { Picante } \\
\text { Característico }\end{array}$ & $\begin{array}{c}\text { Picante } \\
\text { Característico }\end{array}$ & $\begin{array}{c}\text { Picante } \\
\text { Característico }\end{array}$ \\
Califica & Bueno & Bueno & Bueno & Bueno \\
Puntaje & 4 & 4 & 4 & 4 \\
\hline
\end{tabular}

En lo que respecta al análisis sensorial no se encontró diferencia entre el control y las diferentes dosis ensayadas, al evaluarse cuantitativamente, los jueces panelistas calificaron con un promedio de puntaje de 4 sobre una escala hedónica de 1 a 5 , correspondiendo a este puntaje una calificación de BUENO para todos los tratamientos.

\section{CONCLUSIONES}

La muestra presentó una calidad microbiológica deficiente con predominio de microorganismos aerobios mesófilos de $3,7 \times 10^{6} \mathrm{UFC} / \mathrm{g}$ y recuento de hongos de $5 \times 10^{3} \mathrm{UFC} / \mathrm{g}$.

No se encontraron levaduras, coliformes totales, Salmonella/Shiguella, Clostridium sulfito reductor ni Escherichia coli.

Dosis de $7 \mathrm{kGy}$ en páprika en polvo reduce la población de microorganismos de aerobios mesófilos en 5 ciclos logarítmicos, de $3,7 \times 10^{6} \mathrm{UFC} / \mathrm{g}$ a $2,5 \times 10 \mathrm{UFC} / \mathrm{g}$ cumpliendo con las especificaciones requeridas.

Dosis de 4 kGy son suficiente para reducir la

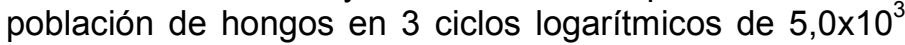
UFC/g a $<10$ UFC/g.

A dosis de $7 \mathrm{kGy}$ las características físico-químicas y la evaluación sensorial no son afectadas significativamente.

De acuerdo a los resultados, la dosis mínima óptima seleccionada que redujo la población microbiana a las especificaciones requeridas, sin alterar las características intrínsecas de la páprika en polvo fue de 7 kGy. 


\section{REFERENCIAS BIBLIOGRÁFICAS}

[1]. Farkas, J.Tratamiento de las Especias por Irradiación. La Irradiación de Alimentos en Latinoamérica IAEA TECDOC 331.Viena. (1985) 123-143.

[2]. Wittig de Penna et al. Evaluación sensorial de pimentón en polvo (Capsicum Annum) irradiado. Nucleotécnica, Santiago de Chile. Año $10 N^{\circ} 18$ (Abril1990) 47-54

[3]. Vivanco, M y Vargas, J. Evaluación de páprika en polvo irradiado. Informe Científico Tecnológico ISSN 1684-1662. Lima, Perú.(2005) 129-131

[4]. Derinat. Páprika. [serie en internet (2007). Disponible en URL : http://www.derinat.com/paprika.htm

[5]. Centrum Católica.Páprika Peruana en el mercado internacional. Reporte Anual (2008). [serie en internet]. Disponible en URL:

http://1.1.1.1/856728632/855818288T081124120043.txt.binXMysM0dapplication/pdfXsysM0dhttp://www.centrum2. pucp.edu.pe/cemaldia/informes_pdf/Ficha_páprika_2008_pdf.

[6]. Documento GCIIA N 5.(1991). Código de las buena practicas de irradiación para el control de patógeno y otra microflora en especias, hierbas y otros sazonadores Vegetales. FAO/OIEA/OMS, Viena.

[7]. Norma ITINTEC 209,239 Ají panca (1984)

[8]. [Código Alimentario Argentino. Resolución Conjunta 92/2008 y 355/2008. (2008). Disponible en URL: $\mathrm{http}: / / \mathrm{www}$. senasa.gov.ar/contenido.php?to=n\&in=1197\&ino=1197\&io=7854

E-mail: jvargas@ipen.gob.pe 